\title{
Úlceras del pie diabético: importancia del manejo multidisciplinario y salvataje microquirúrgico de la extremidad
}

\author{
Nicolás Pereira C. ${ }^{1,2}$, Hyunsuk Peter $\operatorname{Suh}^{3}$ y Joon Pio (JP) Hong ${ }^{3}$
}

\section{Diabetic foot ulcers: importance of a multidisciplinary approach and microsurgical limb rescue}

Diabetic foot ulcers are the main risk factor for non-traumatic amputations in people with diabetes. A succesful intervention requires a thorough understanding of the pathogenesis and a timely and standardized implementation of an effective treatment. A multidisciplinary approach is needed to initially control and treat multiple factors that cause severe diabetic foot ulceration. Debridement, infection control, and revascularization are key steps in wound stabilization and preparation for successful reconstruction. Microsurgery and supermicrosurgery provide a well-vascularized tissue to control infection, an adequate shoe contour, durability and solid anchorage to resist shearing forces during gait. In this way, the salvage of the limb can be achieved, improving the quality of life and increasing the survival rate.

Key words: diabetic foot; multidisciplinary approach; microsurgery; supermicrosurgery.

\section{Resumen}

Las úlceras del pie diabético son el principal factor de riesgo para las amputaciones no traumáticas en personas con diabetes. El éxito de la intervención requiere un completo entendimiento de la patogénesis y una implementación oportuna y estandarizada de un tratamiento efectivo. Es necesario un enfoque multidisciplinario para inicialmente controlar y tratar múltiples factores causantes de la ulceración severa del pie diabético. El desbridamiento, control de la infección y revascularización son pasos clave para la estabilización de la herida y su preparación para una reconstrucción exitosa. La microcirugía y supermicrocirugía permite proporcionar un tejido bien vascularizado para controlar la infección, un contorno adecuado para el calzado, durabilidad y anclaje sólido para resistir las fuerzas de cizallamiento durante la marcha. De esta manera, se puede lograr el salvataje de la extremidad, mejorando la calidad de vida y aumentando la sobrevida.

Palabras clave: pie diabético; manejo multidisciplinario; microcirugía; supermicrocirugía.

\section{Introducción}

\section{Epidemiología}

La prevalencia estimada de diabetes en el mundo es $2,8 \%$ en el año 2000 y se espera que aumente al $4,4 \%$ para el $2030^{1}$. En Chile se calcula que existen 1.372 .700 personas con diabetes, lo que representa el $11 \%$ de la población adulta, posicionando a nuestro país en el segundo lugar de Sudamérica ${ }^{2}$.

El pie diabético presenta una alteración anatómica o funcional, determinada por anomalías neurológicas y/o diversos grados de enfermedad vascular periférica, que le confiere una mayor susceptibilidad a presentar infección, ulceración y/o destrucción de tejidos profundos. Las úlceras del pie diabético son el principal factor de riesgo para las amputaciones no traumática en personas con diabetes. Se estima que aproximadamente el 3-4\% de los diabéticos presenta actualmente alguna úlcera y el $25 \%$ de los pacientes con esta enfermedad desarrollará úlceras en sus pies en algún momento de su vida ${ }^{3}$.

El riesgo de amputación aumenta 8 veces una vez que se presenta una úlcera y se estima que éste es 15 veces mayor en pacientes diabéticos comparado con
'Departamento de Cirugía Plástica, Clínica Las Condes. Santiago, Chile.

${ }^{2}$ Departamento de Cirugía Plástica y Quemados, Hospital del Trabajador. Santiago, Chile.

${ }^{3}$ Department of Plastic Surgery, Asan Medical Center, University of Ulsan College of Medicine. Seoul, Republic of Korea.

Recibido el 22 de octubre de 2017, aceptado para publicación el 11 de diciembre de 2018.

\section{Correspondencia a:} Dr. Nicolás Pereira C. npereira@clc.cl 
personas sanas ${ }^{4}$. La mortalidad a 5 años de pacientes con amputaciones mayores varía de $39 \%$ a $80 \% 0^{3,5}$, por lo tanto, el salvataje del pie diabético es importante debido a que reduce el impacto económico, mejora la calidad de vida y aumenta la sobrevida de los pacientes ${ }^{6}$.

\section{Fisiopatología del pie diabético}

\section{Neuropatía}

El daño neurológico en diabéticos afecta fibras motoras, sensitivas y autonómicas. La combinación de la disfunción motora y sensitiva puede causar un estrés anormal en el pie resultando en trauma. La neuropatía autonómica simpática provoca vasodilatación y disminución de la sudoración, resultando en pies tibios y muy secos que son propensos a heridas, así como a alteraciones funcionales en el flujo microvascular ${ }^{7}$. El pie diabético neuropático no se ulcera espontáneamente, sino más bien en combinación con alguna forma de trauma. La vía causal más común para la ulceración es la suma de neuropatía (pérdida sensitiva), deformidad (ej: cabezas metatarsianas prominentes) y trauma (ej: calzado mal adaptado $)^{7}$.

La neuropatía diabética altera el reflejo del axonal que depende de la función nociceptiva de las fibras-C y provoca vasodilatación local en respuesta a un estímulo doloroso. Este deterioro puede explicar en parte por qué algunas úlceras en el pie diabético neuropático son lentas o fracasan en cicatrizar, a pesar de una adecuada revascularización ${ }^{8}$.

\section{Isquemia}

La enfermedad arterial oclusiva (EAO) se caracteriza por la estenosis y oclusión. Es el resultado de la arterosclerosis avanzada que ocurre en diabéticos en las arterias femorales, poplíteas, tibiales, peroneas y pedias.

Los pacientes con EAO y diabetes son más propensos a desarrollar úlceras isquémicas que los pacientes sin estas enfermedades. A pesar que la mayoría de las úlceras del pie diabético no se asocian a la arterosclerosis de vasos de la pierna y la isquemia subsecuente $^{9}$, es probablemente la principal causa evitable de amputación. Es importante la revascularización temprana en pacientes con úlceras del pie diabético. Incluso si se produce una re-oclusión, la ventaja de proporcionar una revascularización temporal es muy significativa, ya que esto puede ser suficiente para que la herida cicatrice.

\section{Daño microvascular}

Se produce por la hiperglicemia crónica que lleva a un aumento en la actividad de la vía de los polio- les, con incremento de sorbitol y posterior a ello de fructosa, generando estrés oxidativo. Así, aumenta la producción de superóxidos en la mitocondria que inactivan el óxido nítrico y contribuyen a disfunción vascular, impidiendo una correcta reparación y promoción de la angiogénesis, migración y proliferación de fibroblastos, células epiteliales, endoteliales y queratinocitos. Por otro lado, favorece la acumulación de productos de glicación avanzada implicados en la patogénesis de las complicaciones diabéticas incluyendo alteración de la cicatrización de heridas. Esta condición de estrés oxidativo en diabéticos a nivel vascular puede aumentar diacilglicerol y proteína quinasa $\mathrm{C}$, todo lo cual contribuye a mayor disfunción vascular, inflamación e injuria celular ${ }^{10}$.

\section{Evaluación inicial y manejo multidisciplinario}

La amplia manifestación de síntomas del pie diabético se debe a una fisiopatología multifactorial $^{11}$. Estas condiciones pueden actuar de manera independiente o sinérgicamente para dar lugar a deformidades de Charcot, contracturas del tendón de Aquiles, ulceraciones, necrosis y gangrena.

El equipo multidisciplinario, encabezado generalmente por una enfermera, coordina y administra eficientemente los recursos. Su evaluación inicial consiste en hacer un screening de la condición sistémica y de los pies, haciendo énfasis en la historia médica general y específica de la diabetes; antecedentes de la úlcera, episodios previos y factores condicionantes; características de la piel de los pies; antecedentes sociodemográficos, entre otros. Se debe recomendar el uso de calzado adecuado, la evaluación diaria de los pies y consultar ante la aparición de cualquier herida. La hiperqueratosis debe ser removida periódicamente a medida que se vaya formando ya que esto permite disminuir la presión sobre la superficie plantar y la consecuente formación de úlceras. Se debe realizar el tratamiento de la onicomicosis ya que la alteración de las uñas puede provocar sobreinfecciones.

La evaluación inicial del pie debe incluir cuatro categorías: vascular, neurológica, ortopédica e infecciosa (Tabla 1).

Habitualmente, las úlceras del pie diabético se clasifican según Wagner en seis grados ${ }^{12}$. Sin embargo, esta clasificación resulta incompleta ya que incluye la infección solo en un tipo de úlceras y la enfermedad vascular en dos de ellas, teniendo que ser esta última muy severa. Consideramos que clasificación de la Universidad de Texas de San Antonio es más completa ya que combina el grado 
de profundidad (0-III) con la presencia o ausencia de infección/isquemia (A-D) (Tabla 2) ${ }^{13}$.

Luego del screening inicial, el tratamiento comienza con un estricto control de la glicemia y apoyo nutricional, derivando al paciente al endocrinólogo y nutricionista. En caso de existir alteraciones en alguna de las categorías del examen físico, se debe derivar a los especialistas correspondientes para su posterior tratamiento y evaluación. Cuando estamos en presencia de una infección masiva y pérdida de tejido, se debe contactar al cirujano plástico para el desbridamiento inicial de urgencia y la toma de decisiones posteriores. Este es el primer paso y el más importante para limitar la diseminación de la infección y realizar el salvataje del pie diabético ${ }^{14}$.

La interconsulta a cirugía vascular es esencial cuando el paciente es sintomático o frente a una úlcera que no cicatriza. Las úlceras neuropáticas requieren desbridamiento del tejido desvitalizado o infectado, combinado con el cuidado local de la herida y la descarga del pie.

La osteomielitis en el pie diabético se debe manejar mediante resección quirúrgica y tratamiento antibiótico. La tríada radiológica clásica (desmineralización, reacción perióstica y destrucción ósea) se presenta cuando el $30-50 \%$ del hueso se ha destruido, proceso que puede tardar 2 semanas ${ }^{15}$. La resonancia magnética y la biopsia ósea son los métodos diagnósticos de elección para osteomielitis en úlceras del pie diabético. Cuando hay hueso visible en la úlcera, el valor predictivo es superior a $90 \%$ en el diagnóstico de osteomielitis. De igual manera el "bone probe" (golpear el fondo de la úlcera con un estilete romo para sentir el hueso) tiene valor predictivo similar, por lo que si es positivo no se
Tabla 1. Evaluación inicial del pie diabético en las cuatro categorías

\begin{tabular}{|ll|}
\hline Categoría & Evaluación inicial \\
Vascular & $\begin{array}{l}\text { Claudicación o dolor de reposo, pulsos, llene capilar y caracte- } \\
\text { rísticas de la piel y fanéreos }\end{array}$ \\
Neurológica & $\begin{array}{l}\text { Sensibilidad táctil, vibratoria y propioceptiva. Se debe realizar } \\
\text { el test de monofilamente 5.07 (Semmes-Weinstein) para evaluar } \\
\text { la sensibilidad protectora }\end{array}$ \\
Ortopédica & $\begin{array}{l}\text { Prominencias óseas, deformidades de los dedos en martillo, } \\
\text { garra, hallux valgus, pie plano o cavo, deformidad de Charcot, } \\
\text { entre otras }\end{array}$ \\
Infecciosa & $\begin{array}{l}\text { Presencia de úlcera, dolor, fiebre, descarga purulenta, celulitis, } \\
\text { cultivos, presencia de osteomielitis }\end{array}$ \\
\hline
\end{tabular}

necesitan más pruebas para diagnosticar la osteomielitis. El valor predictivo negativo es $56 \%$, por lo que si el examen es negativo se debe confirmar con otros métodos diagnósticos ${ }^{16}$. Se recomienda realizar radiografías de rutina, si se sospecha osteomielitis (úlcera que no cicatriza, palpación cercana al hueso) se deben hacer estudios complementarios como cintigrama óseo, resonancia magnética o bone probe.

Cuando la cicatrización se detiene a pesar de un adecuado cuidado (descarga, control de la infección, control del edema y curaciones avanzadas), se debe considerar la terapia con presión negativa, oxígeno hiperbárico, terapia celular y tratamiento con factor de crecimiento (factor de crecimiento epidérmicoEGF). Un 53\% de disminución del área de la úlcera en 4 semanas es un importante predictor de cicatrización ${ }^{17}$. El progreso de la cicatrización se supervisa y cuando este se vuelve estacionario, a pesar del tratamiento multimodal, se considera indicación para la reconstrucción.

Tabla 2. Clasificación de la Universidad de Texas de San Antonio para las úlceras del pie diabético ${ }^{19}$

\begin{tabular}{|c|c|c|c|c|}
\hline \multicolumn{5}{|c|}{ GRADOS } \\
\hline & 0 & I & II & III \\
\hline A & $\begin{array}{l}\text { Lesión pre o posulcerativa com- } \\
\text { pletamente epitalizada }\end{array}$ & $\begin{array}{l}\text { Úlcera superficial que no } \\
\text { compromete tendones, cápsula } \\
\text { ni hueso }\end{array}$ & $\begin{array}{l}\text { Úlcera que compromete } \\
\text { tendones o cápsula }\end{array}$ & $\begin{array}{l}\text { Úlcera que compromete hueso o } \\
\text { articulación }\end{array}$ \\
\hline B & $\begin{array}{l}\text { Lesión pre o posulcerativa } \\
\text { completamente epitalizada con } \\
\text { infección }\end{array}$ & $\begin{array}{l}\text { Úlcera superficial que no } \\
\text { compromete tendones, cápsula } \\
\text { ni hueso con infección }\end{array}$ & $\begin{array}{l}\text { Úlcera que compromete } \\
\text { tendones o cápsula con } \\
\text { infección }\end{array}$ & $\begin{array}{l}\text { Úlcera que compromete hueso o } \\
\text { articulación con infección }\end{array}$ \\
\hline D & $\begin{array}{l}\text { Lesión pre o posulcerativa } \\
\text { completamente epitalizada con } \\
\text { infección e isquemia }\end{array}$ & $\begin{array}{l}\text { Úlcera superficial que no } \\
\text { compromete tendones, cápsula } \\
\text { ni hueso con infección e } \\
\text { isquemia }\end{array}$ & $\begin{array}{l}\text { Úlcera que compromete } \\
\text { tendones o cápsula con } \\
\text { infección e isquemia }\end{array}$ & $\begin{array}{l}\text { Úlcera que compromete hueso } \\
\text { o articulación con infección e } \\
\text { isquemia }\end{array}$ \\
\hline
\end{tabular}


Figura 1. Algoritmo general para el tratamiento del pie diabético.

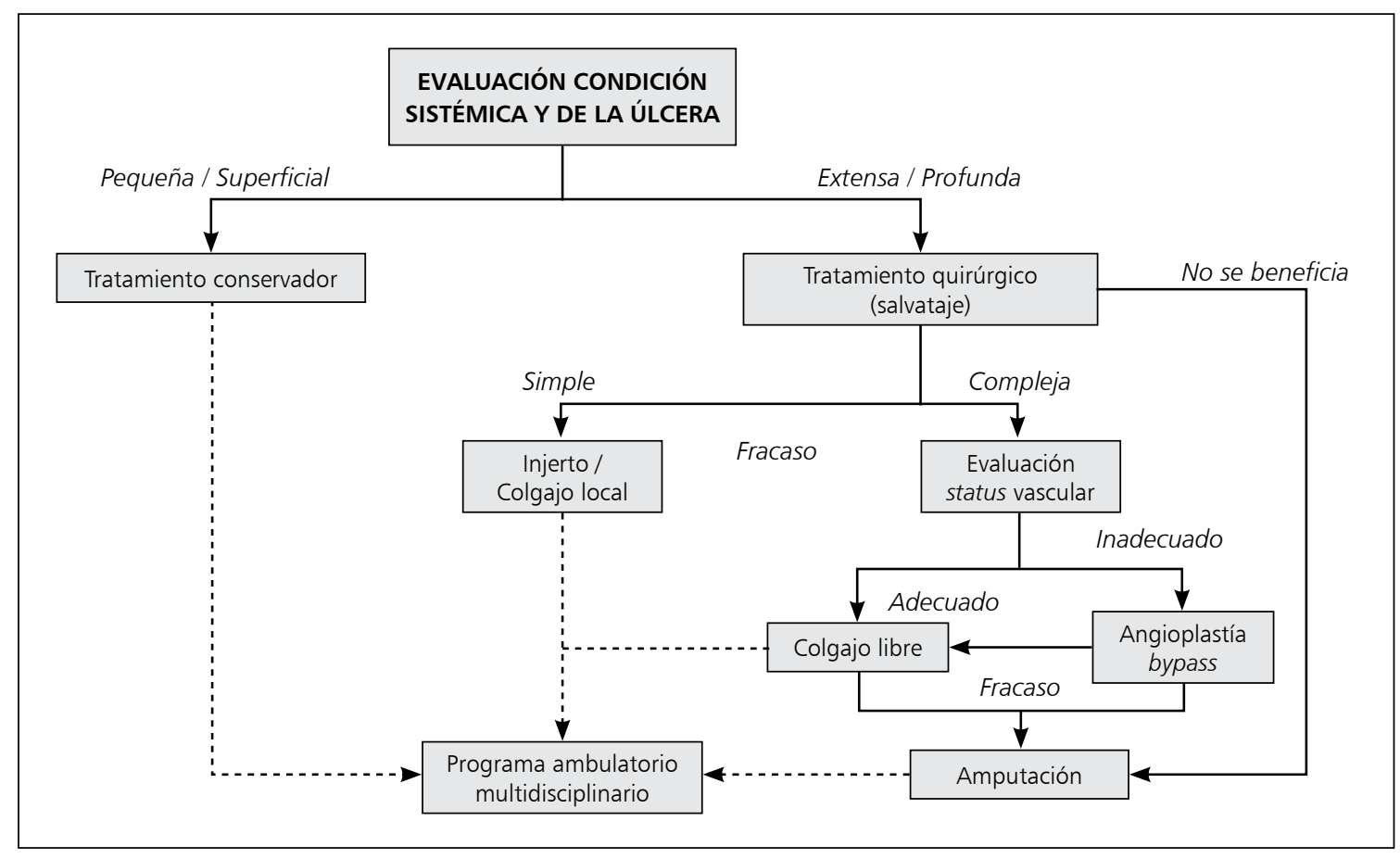

En los centros que realizan un manejo multidisciplinario, la tendencia ha cambiado desde las amputaciones mayores al salvataje de la extremidad ${ }^{18}$. Al considerar la perfusión, infección, tratamiento de úlceras, descarga del pie y cirugía ósea; las tasas de salvataje han aumentado. Una úlcera que no cicatriza no debe ser considerada por sí sola como una indicación de amputación, sino que debe ser estudiada para resolver las condiciones patológicas subyacentes ${ }^{19}$.

\section{Tratamiento quirúrgico inicial}

El manejo quirúrgico comienza con el desbridamiento y el control de la infección. Una vez estabilizado el paciente y la úlcera, se realiza una evaluación adicional de la herida (Figura 1$)^{20}$.

Las úlceras complejas requieren estudio vascular, el cual incluye las siguientes evaluaciones:

- La Angiografía por Tomografía Computada (CTA) se realiza en los pacientes candidatos para una reconstrucción microquirúrgica para evaluar el estado vascular general y de las colaterales (Figura 2). En pacientes diabéticos, los vasos colaterales pueden ser el tronco principal de la extremidad hacia distal, por lo que la elevación de un colgajo basado en estas colaterales, puede causar la isquemia de la misma ${ }^{21}$.
Figura 2. Angiografía por Tomografía Computada donde se observa aterosclerosis de ambas extremidades inferiores, principalmente en las piernas.

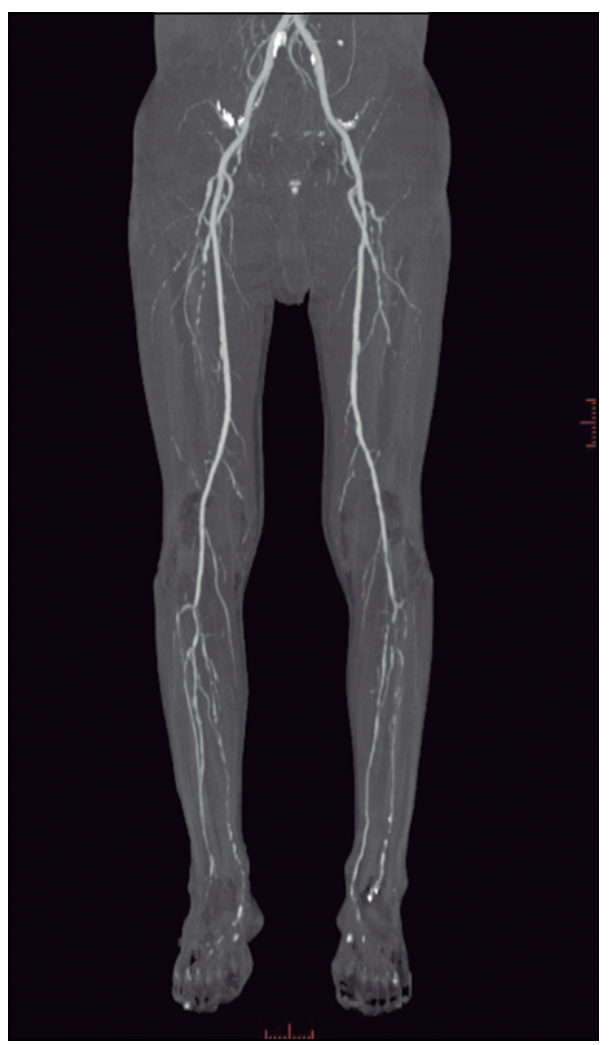


- El doppler ultrasonido se utiliza para confirmar el flujo vascular distal. Proporciona información para la selección de los vasos receptores ya que una velocidad de flujo máximo (peak flow velocity) $>40 \mathrm{~cm} / \mathrm{seg}$ en los vasos receptores se ha asociado a una mayor sobrevida del colgajo.

- La Presión Transcutánea de Oxígeno $\left(\mathrm{TcPO}_{2}\right)$ $>30 \mathrm{mmHg}$ con $\mathrm{O}_{2}$ ambiental es un factor predictivo para el éxito de la cicatrización, mientras que una presión $<30 \mathrm{mmHg}$ es probable que siga un curso desfavorable ${ }^{22}$. Luego de la revascularización, si la úlcera persiste bajo este valor, se indica oxígeno hiperbárico. Si la $\mathrm{TcPO}_{2}$ alrededor de la úlcera es $>30 \mathrm{mmHg}$, se planifica un tratamiento adicional incluyendo procedimientos reconstructivos. De lo contrario, se realiza la amputación de acuerdo al nivel.

- El índice tobillo-brazo (ABI) no se utiliza porque no es confiable en pacientes diabéticos ${ }^{23}$.

Si luego de estas evaluaciones el estado vascular está en duda, se deriva entonces a cirugía vascular para estudio con angiografía y revascularización con angioplastia o bypass.

\section{Desbridamiento}

Evaluar, desbridar y tratar la infección de manera oportuna para evitar amputaciones y hospitalizaciones prolongadas. Todo el tejido blando y óseo desvitalizado e infectados se deben resecar durante el desbridamiento y luego se irriga abundantemente para reducir el recuento bacteriano ${ }^{24}$. Se deben tomar cultivos de tejidos.

Los desbridamientos deben ser seriados como parte de la preparación de la úlcera para la reconstrucción. La proteína $\mathrm{C}$ reactiva se puede utilizar como indicador de infecciones ocultas o posibles infecciones después de la reconstrucción.

Es importante tener conocimiento sobre los angiosomas y su irrigación. En la región del tobillo y pie existen 6 angiosomas irrigados por ramas de las arterias tibial posterior, tibial anterior y peronea ${ }^{25}$. La comprensión de la distribución vascular ayuda a planificar no sólo la reconstrucción, sino también el desbridamiento. Al planificar la reconstrucción con colgajos locales, se debe diseñar de manera que no viole el territorio del angiosoma, lo cual puede provocar la necrosis del colgajo ${ }^{26}$. Además, al realizar el desbridamiento guiado por angiosomas, la sobrevida de los colgajos aumentaría al incrementar la probabilidad de revascularización marginal del angiosoma circundante sano por inosculación vascular.

\section{Salvataje microquirúrgico de la extremidad}

Hasta hace poco se consideraba que los diabéticos tenían una mayor incidencia de enfermedad de los vasos pequeños que resultaba en úlceras en los pies $^{27}$. Sin embargo, estudios posteriores no han logrado demostrar un aumento en la oclusión arteriolar o de la proliferación endotelial ${ }^{28}$. En diabéticos, la enfermedad oclusiva ocurre principalmente en la pierna, de modo que las arterias del pie están menos alteradas. Basados en esto, el salvataje del pie diabético utilizando un enfoque microquirúrgico ha mostrado un éxito similar al de pacientes no diabéticos ${ }^{29,30}$. Se ha reportado una sobrevida de los colgajos de $92 \%$ en pacientes diabéticos y una tasa de rescate de las extremidades de $83,4 \%$ durante un período de seguimiento de 28 meses. Este estudio, además, indica que los colgajos libres en el manejo de las úlceras no traumáticas en pacientes diabéticos pueden evitar amputaciones ${ }^{30}$.

\section{Amputaciones}

Hay casos en que las amputaciones son inevitables. Con mucha frecuencia, las amputaciones se realizan a un nivel más proximal que el requerido para poder tener suficiente cantidad de tejidos blandos y así asegurar el cierre del defecto. El uso de colgajos libres para cubrir defectos de amputaciones parciales es una estrategia eficiente para mantener la longitud del pie y preservar un patrón de marcha cercano al normal ${ }^{31}$. Además, permite evitar amputaciones mayores, luego de las cuales el paciente tiene una mortalidad a 5 años hasta de $80 \% \%^{3,5}$. Se ha reportado que el salvataje de la extremidad mediante microcirugía aumenta la sobrevida a 5 años desde $41,1 \%$ hasta $76,8 \%$ luego de una amputación mayor ${ }^{6}$.

\section{Revascularización}

El momento ideal para la reconstrucción después de la revascularización no está claro. Se ha reportado el salvataje exitoso de la extremidad mediante la reconstrucción con colgajo libre y revascularización simultánea $^{32}$. Sin embargo, la tasa de fallo temprano del bypass dentro de los 30 días es alta. Hemos observado la necrosis parcial y total de colgajos a las 2 o 3 semanas luego de realizar la reconstrucción y revascularización simultánea o diferida en pocos días, lo que sugiere que debería existir un período de estabilización suficiente después de la revascula- 
rización. Si la intervención vascular falla y la úlcera progresa, la amputación está justificada.

\section{Selección de pacientes}

Los defectos extensos y complejos del pie se deben considerar para reconstrucción una vez que se ha conseguido un adecuado desbridamiento y una perfusión razonable. En defectos moderados y grandes la reconstrucción con colgajos libres es la técnica de elección, a menos que exista alguna contraindicación para el procedimiento.

Los pacientes que se consideran candidatos para reconstrucción microquirúrgica son aquellos que poseen $^{33}$ :

- Defecto de la extremidad inferior, que no ha mostrado signos de granulación o cicatrización a pesar del desbridamiento adecuado.

- Ninguna enfermedad sistémica significativa que pueda ser exacerbada por múltiples cirugías y rehabilitación prolongada.

- Capacidad previa de deambulación con el objetivo de restaurar una extremidad funcional.

- Compromiso del paciente con la fisioterapia requerida para regresar a la vida normal.

- Velocidad de flujo máxima $>40 \mathrm{~cm} / \mathrm{seg}$ en la arteria receptora.

\section{Vasos receptores y rol de la supermicrocirugía}

El mayor desafío en la reconstrucción microquirúrgica del pie diabético es encontrar un vaso receptor adecuado. Incluso con buen flujo al pie, la aterosclerosis puede hacer que la anastomosis sea muy difícil. Si se utiliza una arteria principal como receptora, se debe buscar un segmento que no esté afectado por la calcificación. Se recomienda una anastomosis termino-lateral porque los vasos principales pueden estar calcificados y el flujo puede provenir de la tibial anterior o tibial posterior (anterógrado o retrógrado). Se ha reportado el "fenómeno de robo vascular", en el cual el flujo puede ser desviado al lecho vascular de baja resistencia constituido por el colgajo ${ }^{34}$. Debemos recordar que, especialmente para la extremidad isquémica, el reducido aporte vascular es el causante de la úlcera, por lo tanto, se deben hacer todos los esfuerzos para preservar el flujo distal al pie.

Generalmente la zona de isquemia y necrosis coincide con el territorio de un angiosoma ${ }^{25,26}$. Durante el período de transición a la isquemia del pie, se desarrollan vasos colaterales desde los an- giosomas adyacentes para asegurar una irrigación alternativa. El uso de estas pequeñas arterias del angiosoma adyacente sano como vasos receptores extiende las posibilidades de reconstrucción en pacientes con pie diabético e isquemia severa. Por otro lado, las ramas de las arterias tibial posterior y pedia excepcionalmente se calcifican, por lo tanto, la anastomosis a estas ramas es más fácil porque se trata de una arteria blanda y flexible, sin disminuir el flujo distal. Un vaso receptor pequeño con buen flujo pulsátil es adecuado como receptor. Por lo tanto, una contraindicación absoluta sería la ausencia de flujo distal al pie, sin signos de perfusión de vasos pequeños.

La supermicrocirugía es una técnica definida como la anastomosis de vasos con diámetro menor a $0,8 \mathrm{~mm}^{35}$. En el caso del pie diabético, permite basar las reconstrucciones en pequeñas ramas de las arterias principales o en perforantes sin aterosclerosis cuando no existen vasos mayores disponibles. Además, se evita la necesidad de disección de largos pedículos y, por lo tanto, disminuye la morbilidad de la zona dadora. Desempeña un papel alternativo en la reconstrucción del pie diabético ya que permite que la anastomosis a un pequeño vaso del angiosoma adyacente bien vascularizado proporcione suficiente perfusión para permitir que un colgajo libre cubra el territorio isquémico y otorgue una adecuada irrigación ${ }^{36}$. Actualmente, el abordaje propuesto en la reconstrucción de las úlceras del pie diabético consiste en utilizar el concepto de angiosomas y supermicrocirugía free style $e^{37}$. Este enfoque requiere una habilidad refinada y un cambio de paradigma en la reconstrucción.

\section{Colgajos}

Los colgajos para la reconstrucción del pie diabético deben proporcionar un tejido bien vascularizado para controlar la infección, aportar un contorno adecuado para el calzado, ser duraderos y poseer un anclaje sólido para resistir las fuerzas de cizallamiento. Es controversial si los colgajos musculares con injerto de piel, los colgajos fasciocutáneos o los colgajos de perforantes otorgan una solución óptima en la reconstrucción del pie especialmente en las zonas de apoyo. Sin embargo, mientras el defecto esté cubierto con tejido bien vascularizado, proporcionará un suministro vascular independiente para erradicar la infección y aumentar el aporte de oxígeno, incrementando la efectividad antibiótica y la neovascularización del tejido isquémico adyacente ${ }^{38}$. 
Hoy en día, existe una tendencia hacia el uso de colgajos de perforantes, como el anterolateral de muslo (ALT) ${ }^{29}$ (Figura 3), el colgajo de perforante glútea $^{39}$ y el colgajo de perforante de la arteria circunfleja ilíaca superficial (SCIP) ${ }^{40}$ (Figura 4). Estos colgajos proporcionan una cobertura delgada para minimizar el cizallamiento ya que se puede elevar incluyendo sólo la grasa superficial para imitar los septos fibrosos de la planta del pie para adherirse firmemente. Además, mejoran la neovascularización del plexo subdérmico con tejido adyacente y proporciona un adecuado aporte sanguíneo para combatir la infección.

\section{Conclusiones}

Las úlceras del pie diabético son frecuentes y sin el tratamiento adecuado pueden terminar en amputación. El éxito de la intervención requiere un completo entendimiento de la patogénesis de las úlceras del pie diabético y una implementación rápida y estandarizada de un tratamiento efectivo. El manejo multidisciplinario y la reconstrucción microquirúrgica pueden lograr el salvataje de la extremidad, que conducirá a una mejor calidad de vida y sobrevida.

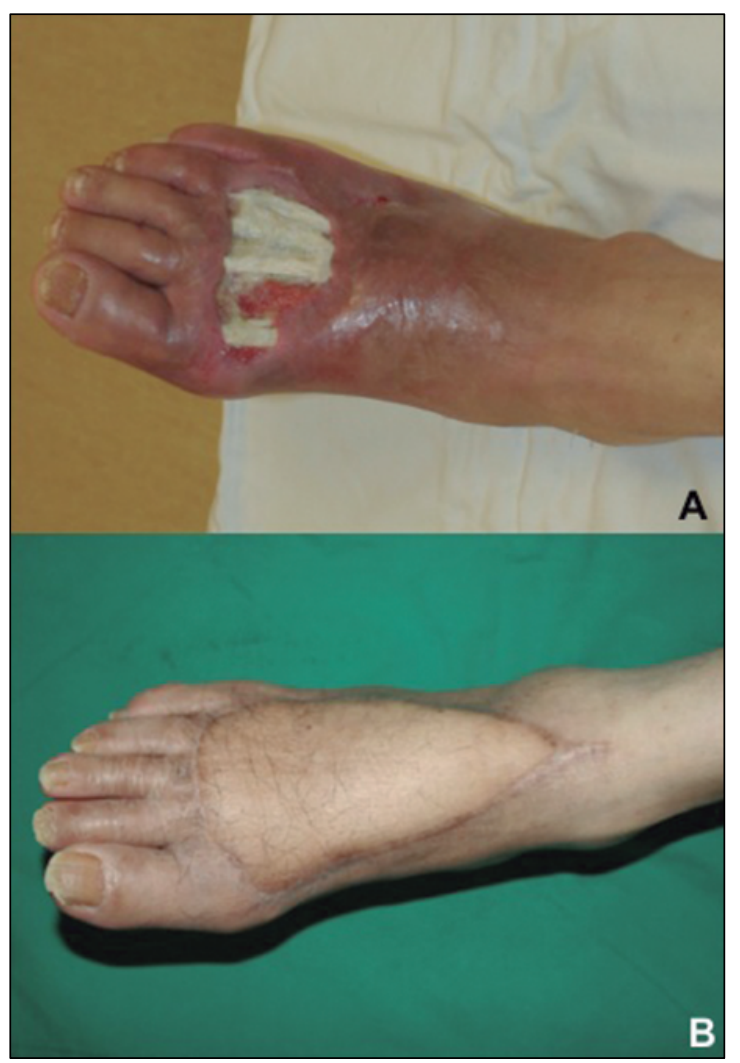

Figura 3. Paciente de 60 años con úlcera en el dorso del pie derecho reconstruido con colgajo ALT mediante supermicrocirugía reemplazando el angiosoma comprometido. A) Imagen preoperatoria; B) Imagen a los 12 meses posoperatorio.

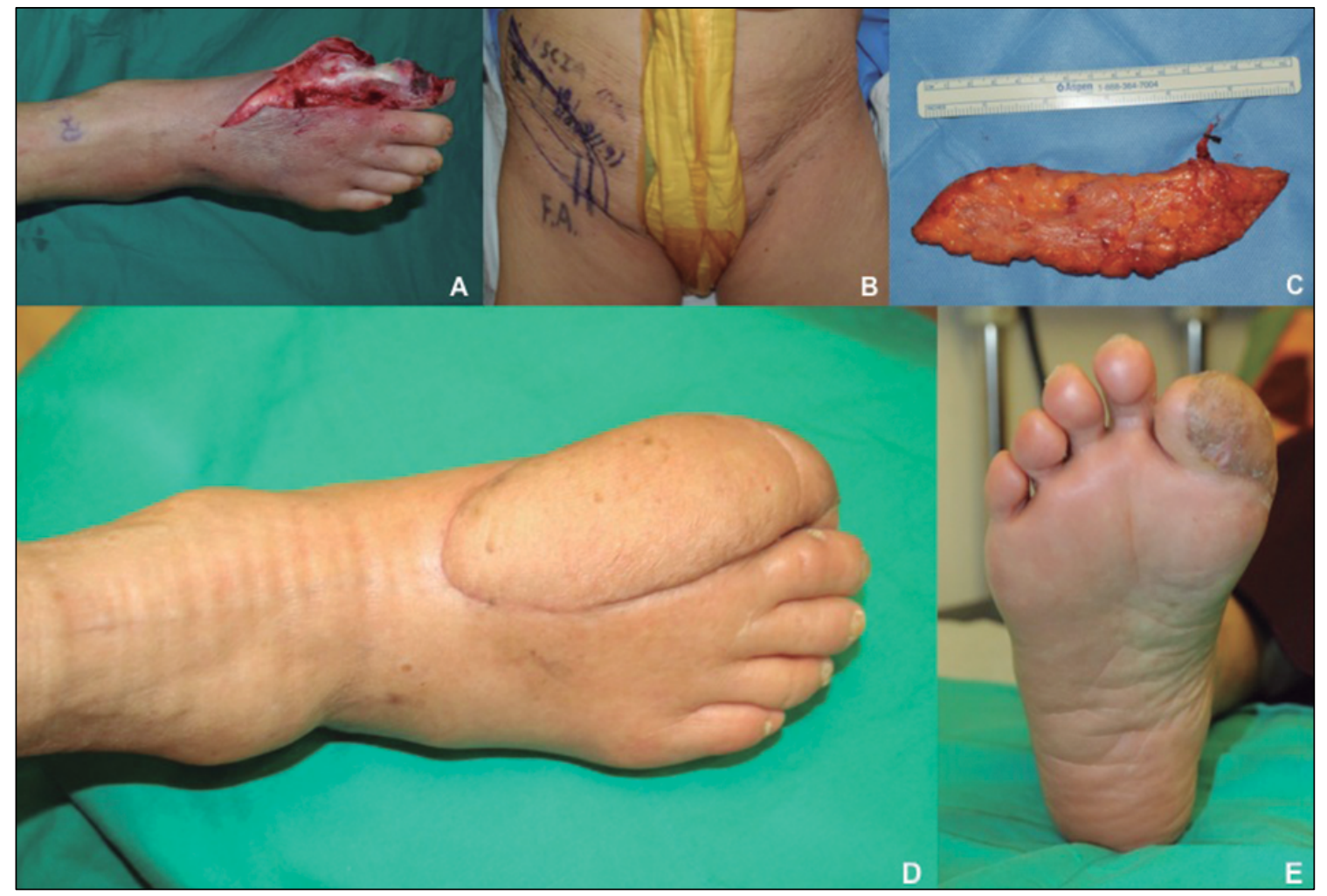

Figura 4. Paciente de 63 años con úlcera en el primer ortejo del pie derecho: A) Imagen intraoperatoria luego del desbridamiento; B) Diseño de colgajo SCIP en la región inguinal derecha; C) Colgajo de SCIP; D-E) Imágenes a los 14 meses posoperatorio. 


\section{Responsabilidades éticas}

Protección de personas y animales. Los autores declaran que para esta investigación no se han realizado experimentos en seres humanos ni en animales.

Confidencialidad de los datos. Los autores declaran que han seguido los protocolos de su centro de trabajo sobre la publicación de datos de pacientes.

Derecho a la privacidad y consentimiento informado. Los autores han obtenido el consentimiento informado de los pacientes y/o sujetos referidos en el artículo. Este documento obra en poder del autor de correspondencia.

Conflicto de intereses: No hay.

\section{Referencias}

1. Wild S, Wild S, Roglic G, Green A, Sicree R, King H. Global prevalence of diabetes: Estimates for the year 2000 and projections for 2030. Diabetes Care 2004;27:1047-53.

2. International Diabetes Federation 2015. www.idf.org/diabetesatlas.

3. Reiber GE. The epidemiology of diabetic foot problems. Diabet Med. 1996;13 Suppl 1:S6-11.

4. Most RS, Sinnock P. The epidemiology of lower extremity amputations in diabetic individuals. Diabetes Care 1983;6:87-91.

5. Moulik PK, Mtonga R, Gill GV. Amputation and mortality in new-onset diabetic foot ulcers stratified by etiology. Diabetes Care 2003;26:491-4.

6. Oh TS, Lee HS, Hong JP. Diabetic foot reconstruction using free flaps increases 5-year-survival rate. J Plast Reconstr Aesthet Surg. 2013;66:243-50.

7. Mayfield JA, Reiber GE, Sanders LJ, Janisse D, Pogach LM. American Diabetes Association. Preventive foot care in people with diabetes. Diabetes Care. 2003;26 Suppl 1:S78-9.

8. Arora S, Pomposelli F, LoGerfo FW, Veves A. Cutaneous microcirculation in the neuropathic diabetic foot improves significantly but not completely after successful lower extremity revascularization. J Vasc Surg. 2002;35:501-5.

9. McDaniel MD, Cronenwett JL. Basic data related to the natural history of intermittent claudication. Ann Vasc Surg. 1989;3:273-7.

10. Nishikawa T, Edelstein D, Du XL, Yamagishi S, Matsumura T, Kaneda Y, et al. Normalizing mitochondrial superoxide production blocks three pathways of hyperglycaemic damage. Nature
2000;404:787-90.

11. Boulton AJ. The diabetic foot: from art to science. The 18th Camillo Golgi lecture.

Diabetologia. 2004;47:1343-53.

12. Wagner FW. The dysvascular foot: a system for diagnosis and treatment. Foot Ankle 1981;2:64-122.

13. Lavery LA, Armstrong DG, Harkless LB. Classification of diabetic foot wounds. J Foot Ankle Surg. 1996;35:528-31.

14. Childers BJ, Potyondy LD, Nachreiner R, Rogers FR, Childers ER, Oberg KC, et al. Necrotizing fasciitis: a fourteen-year retrospective study of 163 consecutive patients. Am Surg. 2002;68:109-16.

15. Tomas MB, Patel M, Marwin SE, Palestro CJ. The diabetic foot. Br J Radiol. 2000;73:443-50.

16. Schinabeck MK, Johnson JL. Osteomyelitis in diabetic foot ulcers: Prompt diagnosis can avert amputation. Postgrad Med. 2005;118:11-5.

17. Sheehan P, Jones P, Giurini JM, Veves A. Percent change in wound area of diabetic foot ulcers over a 4-week period is a robust predictor of complete healing in a 12-week prospective trial. Plast Reconstr Surg 2006;117 Suppl 7:239S-44S.

18. Chiu CC, Huang CL, Weng SF, Sun LM, Chang YL, Tsai FC. A multidisciplinary diabetic foot ulcer treatment programme significantly improved the outcome in patients with infected diabetic foot ulcers. J Plast Reconstr Aesthet Surg. 2011;64:867-72.

19. Cavanagh PR, Ulbrecht JS, Caputo GM. The non-healing diabetic foot wound: fact or fiction? Ostomy Wound Manage 1998;44 Suppl 3A:6S-12S.

20. Knox KR, Datiashvili RO, Granick MS. Surgical wound bed preparation of chronic and acute wounds. Clin Plast Surg. 2007;34:633-41.

21. Suh HP, Kim Y, Hong JP. Multidetector
Computed Tomography (CT) Analysis of 168 Cases in Diabetic Patients with Total Superficial Femoral Artery Occlusion: Is It Safe to Use an Anterolateral Thigh Flap without CT Angiography in Diabetic Patients? J Reconstr Microsurg. 2017 Sep 13. doi: $10.1055 / \mathrm{s}-0037-1606340$.

22. Got I. Transcutaneous oxygen pressure (TcPO2): advantages and limitations. Diabetes Metab. 1998;24:379-84.

23. Goss DE, de Trafford J, Roberts VC, Flynn MD, Edmonds ME, Watkins PJ. Raised ankle/brachial pressure index in insulin-treated diabetic patients. Diabet Med. 1989;6:576-8.

24. Badia JM, Torres JM, Tur C, Sitges-Serra A. Saline wound irrigation reduces the postoperative infection rate in guinea pigs. J Surg Res. 1996;63:457-9.

25. Clemens MW, Attinger CE. Angiosomes and wound care in the diabetic foot. Foot Ankle Clin. 2010;15:439-64.

26. Attinger $\mathrm{C}$, Cooper $\mathrm{P}$, Blume $\mathrm{P}$, Bulan E. The safest surgical incisions and amputations applying the angiosome principles and using the Doppler to assess the arterial-arterial connections of the foot and ankle. Foot Ankle Clin. 2001;6:74599.

27. Goldenberg S, Alex M, Joshi RA. Non atheromatous peripheral vascular disease of the lower extremity in diabetes mellitus. Diabetes 1959;8:261-73.

28. LoGerfo FW, Coffman JD. Current concepts. Vascular and microvascular disease of the foot in diabetes. Implications for foot care. N Engl J Med. 1984;311:1615-9.

29. Hong JP. Reconstruction of the diabetic foot using the anterolateral thigh perforator flap. Plast Reconstr Surg. 2006;117:1599-608.

30. Fitzgerald O'Connor EJ, Vesely M, Holt PJ, Jones KG, Thompson MM, 
Hinchliffe RJ. A systematic review of free tissue transfer in the management of non-traumatic lower extremity wounds in patients with diabetes. Eur J Vasc Endovasc Surg. 2011;41:391-9.

31. Pereira N, Suh Y, Jeon DN, Hong JP, Suh HS. The longer the better: Free flap reconstruction for toe resurfacing and after toes amputation. An effective approach to maintain the length in diabetic foot. Submitted for publication.

32. Randon C, Jacobs B, De Ryck F, Van Landuyt K, Vermassen F. 15-year experience with combined vascular reconstruction and free flap transfer for limb-salvage. Eur J Vasc Endovasc Surg. 2009;38:338-45.

33. Hong JP, Oh TS. An Algorithm for Limb
Salvage for Diabetic Foot Ulcers. Clin Plastic Surg. 2012;39:341-352.

34. Sonntag Murphy RX Jr, Chernofsky MA, Chowdary RP. Microvascular steal phenomenon in lower extremity reconstruction. Ann Plast Surg. 1995;34:336-39 (discussion: 9-40).

35. Koshima I, Yamamoto T, Narushima M, Mihara M, Iida T. Perforator flaps and supermicrosurgery. Clin Plast Surg. 2010;37:683-89, vii-iii.

36. Suh HS, Oh TS, Hong JP. Innovations in diabetic foot reconstruction using supermicrosurgery. Diabetes Metab Res Rev. 2016;32 Suppl 1:275-80.

37. Suh HS, Oh TS, Lee HS, Lee SH, Cho YP, Park JR, Hong JP. A New Approach for Reconstruction of Diabetic Foot
Wounds Using the Angiosome and Supermicrosurgery Concept. Plast Reconstr Surg. 2016;138:702e-9e.

38. Shestak KC, Hendricks DL, Webster MW. Indirect revascularization of the lower extremity by means of microvascular freemuscle flap-a preliminary report. J Vasc Surg. 1990;12:581-5.

39. Hong JP, Yim JH, Malzone G, Lee KJ, Dashti T, Suh HS. The thin gluteal artery perforator free flap to resurface the posterior aspect of the leg and foot. Plast Reconstr Surg. 2014;133:1184-91.

40. Goh TL, Park SW, Cho JY, Choi JW, Hong JP. The search for the ideal thin skin flap: superficial circumflex iliac artery perforator flap-a review of 210 cases. Plast Reconstr Surg. 2015;135:592-601. 